\title{
Simulations for the generation and extraction of negative hydrogen ions in RF-driven ion sources
}

\author{
R. Gutser, D. Wünderlich, U. Fantz, P. Franzen, B. Heinemann, R. \\ Nocentini and the NNBI Team
}

Max-Planck-Institut für Plasmaphysik, EURATOM Association, Boltzmannstr. 2, D-85748

Garching, Germany

\begin{abstract}
The injection of energetic neutral hydrogen atoms plays an important part for plasma heating in fusion experiments. In order to fulfill the requirements of the ITER neutral beam injection (NBI), a RF-driven ion source based on the generation of negative ions prior to neutralization has been successfully developed at IPP Garching. Negative hydrogen ions are generated on a cesiated converter surface (plasma grid) by neutral particles and positive ions and are then transported to the extraction apertures, where the ion beam formation process takes place. Numerical models are necessary to include the relevant physical aspects of these processes. The Monte Carlo transport code CSFLOW is used to describe the dynamical behavior of the cesium distribution on the source walls during vacuum operation. The negative ion transport process is simulated by means of the probabilistic ion transport code TRAJAN, focussing on the effects of aperture diameter variations in mono- and multiaperture extraction systems. A simulation of ion beam formation is carried out with the KOBRA3 ray tracing code, which allows a full $3 \mathrm{~d}$ potential solution without any symmetry restriction. This is necessary to simulate beam steering effects by a non-axisymmetric magnetic field and electrode configuration for the design of the extraction systems for future negative ion experiments.
\end{abstract}

Keywords: Ion Optics, Steering, Particle Orbit and Trajectory, Cesium Flow, Negative Ion Sources PACS: 29.25.Ni, 29.27.Ac, 52.65.Pp, 52.65.Cc, 51.10.+y

\section{INTRODUCTION}

Future fusion devices like ITER require high performance ion sources based on negative hydrogen ions to provide powerful heating and current drive capabilities. The ion source used for ITER should deliver an accelerated $\mathrm{D}^{-}$beam current of $40 \mathrm{~A}$ with a current density of $200 \mathrm{~A} / \mathrm{m}^{2}$ at a source pressure of $0.3 \mathrm{~Pa}$ while maintaining an electron to ion ratio $<1[1]$. The RF-driven ion source was chosen to be the ITER reference source and fulfils the physical aspects of these requirements.

Detailed information regarding the individual components and operation parameters are available from [2]. Figure 1 gives a schematic overview of the source, which is divided into three parts: driver, expansion region and extraction system. A low pressure and low temperature plasma is generated by $1 \mathrm{MHz}$ RF coils with a typical power of $100 \mathrm{~kW}$. The plasma flow enters the expansion region where a magnetic filter field slows down the hot plasma electrons. A typical electron temperature of $2 \mathrm{eV}$ and an electron density of $5 \cdot 10^{17} \mathrm{~m}^{-3}$ is obtained in the vicinity of the extraction system [3]. Negative ion production in the RF-driven ion source is dominated by the surface effect: positive and neutral hydrogen plasma particles are converted on a surface into negative ions by picking up one or more electrons. An enormous increase of the conversion rate can be 


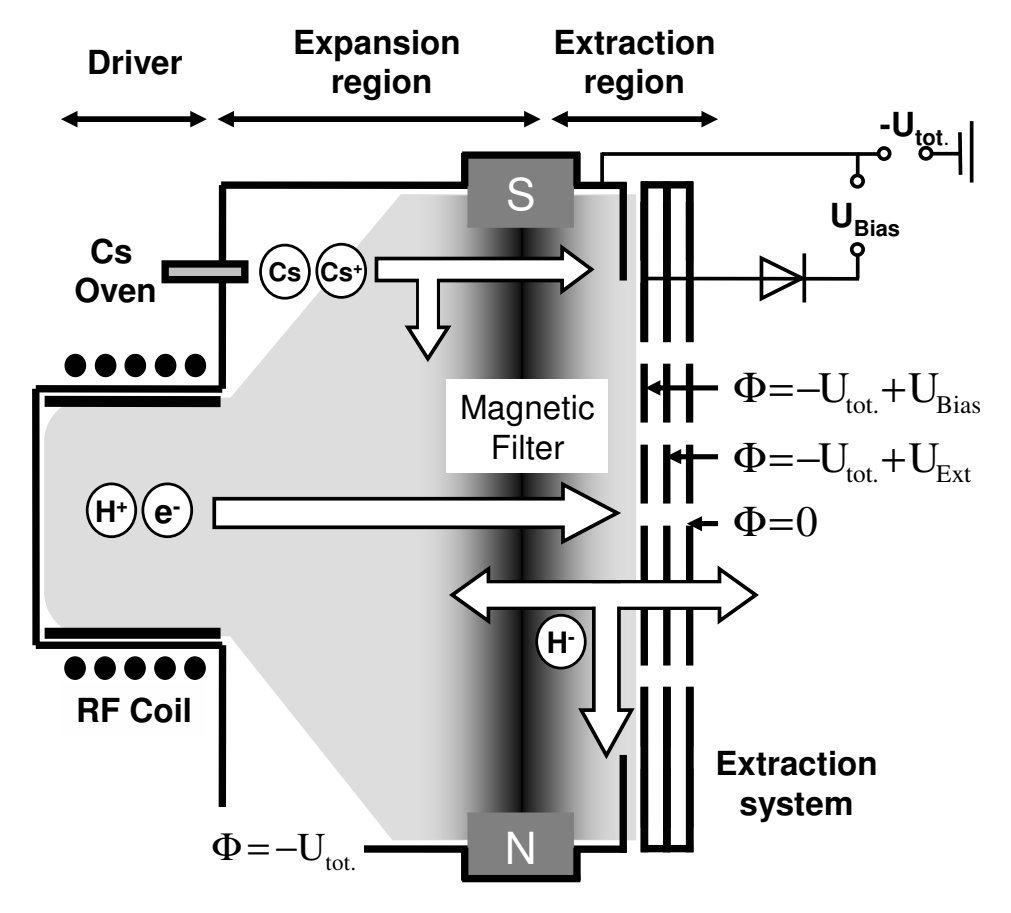

FIGURE 1. Schematic view of the RF-driven ion source. Typical operation parameter are: $\mathrm{U}_{\mathrm{Ext}}=9-10 \mathrm{kV}, \mathrm{U}_{\text {tot. }}=25 \mathrm{kV}$ and $\mathrm{U}_{\text {Bias }} \approx 20 \mathrm{~V}$.

achieved by lowering the work function of the converter material by a coverage with cesium [4], which is evaporated from an oven with a liquid Cs reservoir connected to the back flange of the source body.

Negative ions, which were generated at the surface of the plasma grid, are accelerated towards the plasma by the sheath potential, which is influenced by the bias voltage. This voltage is applied between the first grid of the extraction system and the source body. The negative ions heading away from the extraction system are either destroyed by collisions with plasma particles or redirected into the extraction apertures. These ions are immediately accelerated by the extraction voltage and the beam formation takes place.

Beam formation is accomplished in two stages by a three grid, multiaperture extraction system, which consists of the plasma grid, the extraction grid and the grounded grid. In the first step a voltage of $9-10 \mathrm{kV}$ is applied between the plasma and the extraction grid in combination with a mass selective magnetic filter system to remove co-extracted electrons at reasonable energies. A second voltage of $20-30 \mathrm{kV}$ is currently used for acceleration during the source development, while a stepwise $1 \mathrm{MV}$ acceleration will be used for the ITER NBI.

An understanding of the complex physics of the generation and extraction of negative ions is essential for the further optimization of the source. The aim of this paper is to present three numerical models which can improve the insight in these effects. 


\section{CESIUM 3D TRANSPORT CODE}

An efficient generation of negative ions on the plasma grid converter surface strongly depends on the condition of the Cs layer at the converter surface. The Cs layers on the inner walls of the ion source show a dynamical behavior and Cs redistribution inside the source takes place. As a result, the time scale of the ion source reaching high performance is strongly correlated to the time scale of Cs redistribution. An understanding of the time dependence and spatial distribution of the Cs, especially on the plasma grid, is therefore highly desirable.

The development of the Cs transport code CSFLOW is in progress. It calculates the Cs dynamics during vacuum and plasma operation of the negative ion source. Because of the low ionization energy of $3.89 \mathrm{eV}$, the $\mathrm{Cs}$ is ionized to a large amount during plasma operation. Therefore, neutral Cs particles have to be considered in the vacuum phase, whereas both $\mathrm{Cs}^{+}$ions and neutral Cs have to be treated during plasma operation. The current version of the code considers the Cs distribution for an evacuated source.

During the vacuum operation Cs redistribution is governed by thermal desorption processes from the source walls [5]. The atomic desorption rate of Cs is a sensitive parameter in the simulation. Results of the code using desorption rates of bulk Cs available in literature [6] and the temperature conditions inside the ion source indicate a very rapid loss of the Cs. The resulting depletion of the Cs from the source within the time scale of several hours is in total disagreement with experimental observation of Cs enhanced source operations.

As a result, an accompanied experimental investigation to determine the Cs desorption rate for the conditions of the ion source (background pressure and surface material) is in progress. Cs is evaporated into a small vacuum chamber and the time dependency of the Cs coverage on a coated quartz micro balance is measured for different temperatures under the relevant vacuum conditions of $\mathrm{p}=10^{-5}$ mbar background pressure. The results of this studies indicate a desorption rate, which is reduced by a factor of 50 related to the literature value.

\section{Simulation Model}

The Cs flow from the oven into the evacuated ion source can be considered as a rarefied gas flow. While the oven and the plasma grid are heated to a typical temperature of $150{ }^{\circ} \mathrm{C}$, the source walls are kept at $50{ }^{\circ} \mathrm{C}$. The Knudsen number

$$
K n=\frac{\lambda_{\mathrm{Cs}}}{L}
$$

which is a characteristic quantity for the flow regime, is defined by the mean free path length $\lambda_{\mathrm{Cs}}$ and system dimension $L$. The low background pressure $\left(\mathrm{p}=10^{-5} \mathrm{mbar}\right)$ in the source volume results in a $K n \gg 1$, which is a requirement to treat the Cs in the volume as a free molecular flow. Therefore, collisions of Cs atoms with other gas particles inside the ion source volume can be neglected. As a result, the trajectories of desorbed Cs atoms inside the source follow straight lines. Wall interactions are modeled by a 
cosine distribution of the Cs velocity vector with respect to the surface normal vector. A cartesian surface mesh is used to determine the amount of accumulated Cs on the source walls. Cesium atoms, which enter the aperture area of the extraction system are lost and therefore removed from the simulation.

\section{Simulation Results: Cesium Vacuum Distribution}
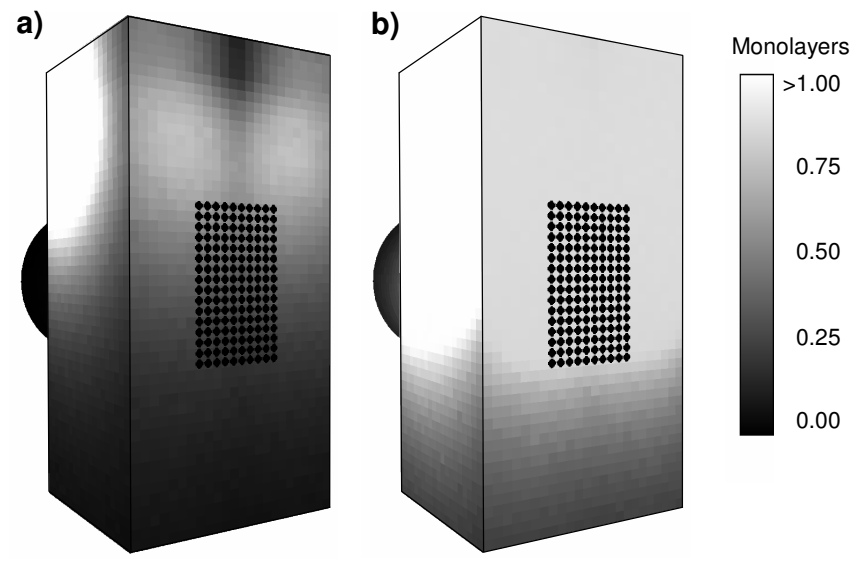

FIGURE 2. Contour plot of the $\mathrm{Cs}$ distribution on the source walls for $50^{\circ} \mathrm{C}$ wall temperature and $150^{\circ} \mathrm{C}$ plasma grid temperature. For a) 5 min Cs evaporation and for b) 20 min evaporation with focus on the Cs distribution on the plasma grid $(<$ $1 \mathrm{ML})$. (Colored version online)

A flow calculation of the Cs within the full 3d geometry of the RF-driven ion source was done for the desorption rate from the experimental investigation and $10 \mathrm{mg} / \mathrm{h} \mathrm{Cs}$ evaporation from the oven [2]. The unit monolayer ML is a convenient description of the Cs wall coverage, where $1 \mathrm{ML}$ corresponds to a Cs layer of $4.5 \cdot 10^{14}$ Atoms $/ \mathrm{cm}^{2}$. Figure 2 a) shows the distribution of $\mathrm{Cs}$ on the side walls of the ion source after 5 minutes of Cs evaporation. Cesium accumulations of several ML are formed on the side walls of the ion source in the vicinity of the oven. Because of Cs desorption from the areas of high Cs accumulation, a Cs re-distribution between the inner walls takes place. Owing to the elevated temperature of the front wall, layer formation on the plasma grid is inhibited and the coverage is limited below $1 \mathrm{ML}$. The Cs coverage of the plasma grid is illustrated by figure $2 \mathrm{~b}$ ), indicating a complete coverage of the aperture area after 20 minutes evaporation under vacuum conditions.

A comparison of the results with the Cs distribution actually observed after opening the ion source indicates, that a Cs distribution with high accumulations on the side walls in the vicinity of the oven, as observed in the simulation, was present in the experiment for (low) wall temperatures of $20{ }^{\circ} \mathrm{C}$ [2]. An elevation of the wall temperatures to $50{ }^{\circ} \mathrm{C}$ resolved this problem in the experiment and resulted in a more homogeneous Cs distribution.

While there is still a discrepancy regarding the temperature dependence of the desorption 
rate, the Cs transport code allows a realistic treatment of the Cs distribution inside the ion source. The validation of thick Cs layers by the computer simulation indicates a significant lower desorption rate of the Cs on the walls of the ion source than expected from literature values of bulk desorption. This effect can be explained by deviations of the layer from bulk Cs due to surface impurity compounds like $\mathrm{CsOH}$ and $\mathrm{CsH}$ resulting in an inhibited desorption.

A parameter variation of the desorption rate is in progress to reproduce the response of the ion source for altered wall-temperature conditions. The further development of the code will also consider effects related to the influence of the plasma on the Cs redistribution.

\section{NEGATIVE ION TRANSPORT CODE}

The distribution of Cs on the plasma grid enables an efficient conversion of positive ions and neutral particles to negative ions. A transport process from the converter surface to the extraction system has to take place in order to extract a negative ion beam. This involves a redirection of the ion velocity vector. The low binding energy $(0.75 \mathrm{eV})$ of the addition electron, which is beneficial to neutralization, is a disadvantage for the transportation process. A theoretical description of the negative ion transport is therefore very important to optimize the ion current delivered by the source.

The geometry of the plasma grid converter, especially the diameter of the extraction apertures, is a question of particular importance. As a consequence, the probabilistic ion transport code TRAJAN was applied to investigate the effect of a variation of the aperture diameter on ion transport for mono- and multiaperture extraction systems.

\section{Simulation Model}

While a detailed description of the TRAJAN code is available from [7], a short summary of the code is given in the following paragraph. The transport code is based on the solution of the Lorentz equation for a statistically significant ensemble of independent test particles with different initial conditions, similar to the model described in [8]. The ions are started from the converter with an energy which represents the acceleration by the sheath voltage. A normal distribution of the spatial coordinates on the converter surface and a cosine velocity distribution with respect to the surface normal vector was chosen for the initial ion state [9]. The electrical fields of plasma sheath and the plasma-beam interface were considered in the simulation, while a zero electrical field was assumed in the plasma volume. Elastic and inelastic collisions of negative ions with plasma particles were simulated using Monte Carlo methods. A binary collision model [10] was used to treat elastic Coulomb collisions with $\mathrm{H}^{+}$particles. The probability of an inelastic collision was calculated by the path length estimator algorithm [11] including the relevant processes listed in table 1 . The calculation of these probabilities requires knowledge of particle densities and temperatures, which were taken from experimental data [12]. The quasi-neutrality condition $n_{\mathrm{H}^{+}}+n_{\mathrm{Cs}^{+}}=n_{\mathrm{e}}$ was used for the calculation. Negative ions are either destroyed by inelastic collisions with the plasma background or 
TABLE 1. Atomic processes taken into account in the transport simulation.

\begin{tabular}{lll}
\hline Electron Stripping & $\mathrm{H}^{-}+\mathrm{e} \rightarrow \mathrm{H}+2 \mathrm{e}$ & {$[13]$} \\
Mutual Neutralization & $\mathrm{H}^{-}+\mathrm{H}^{+} \rightarrow 2 \mathrm{H}$ & {$[14]$} \\
& $\mathrm{H}^{-}+\mathrm{Cs}^{+} \rightarrow \mathrm{H}+\mathrm{Cs}$ & {$[15]$} \\
Charge Exchange & $\mathrm{H}^{-}+\mathrm{H} \rightarrow \mathrm{H}+\mathrm{H}^{-}$ & {$[16]$} \\
\hline
\end{tabular}

extracted by entering the plasma beam interface, while ions hitting the converter electrode are specularly reflected inside the plasma sheath.

An ion transport investigation of the converter geometry was done focussing on the effects of changes of the aperture diameter. For the sake of comparability an unidirectional, constant magnetic field of $8 \mathrm{mT}$ was chosen.

\section{Simulation Results: I. Monoaperture Extraction Systems}
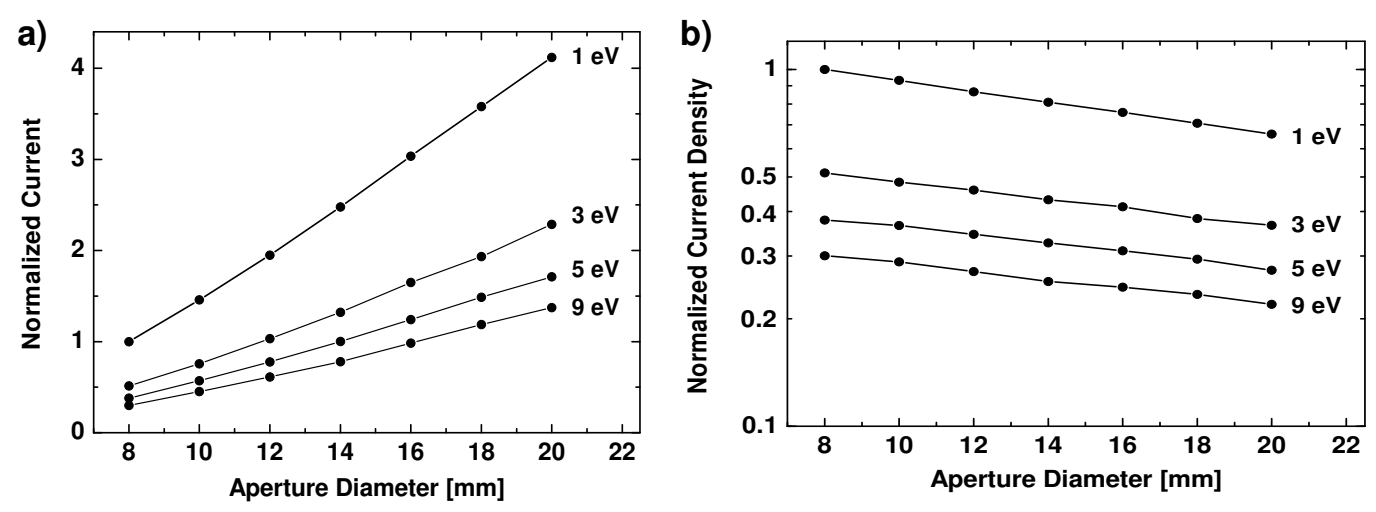

FIGURE 3. a) Normalized aperture current and b) current density versus the aperture diameter for different starting energies and a constant magnetic field of $8 \mathrm{mT}$.

Transport studies for $45^{\circ}$ chamfered monoaperture systems with different aperture diameters $(8 \mathrm{~mm}-20 \mathrm{~mm})$, but constant thickness of $2 \mathrm{~mm}$ were carried out. A computation domain of $5 \times 5 \mathrm{~cm}^{2}$ and a unidirectional filter field of $8 \mathrm{mT}$ was used for the calculation. The number of extracted ions for different aperture diameters and starting energies was normalized with respect to the result for an extraction with $1 \mathrm{eV}$ starting energy and $8 \mathrm{~mm}$ aperture diameter.

Figure 3 a) shows the dependency of the calculated extracted ion current on the aperture diameter for various starting energies. The amount of extracted ions is strongly influenced by the initial energies. Low starting velocities result in a higher retention time of the ions near the plasma grid, which enhances the probability of an extraction process. The variation of the converter geometry manifests its influence in a direct proportionality of the extracted ion current to the aperture diameter. This effect can be explained by the linear correlation between the aperture diameter and the circumferential area en- 
circling the aperture (effective production area). Ions, which are started on this inclined area, have a higher probability to be extracted.

Figure $3 \mathrm{~b}$ ) shows the corresponding dependency of the current density, where the linear behavior of the ion current is exceeded by the quadratic rise of the aperture area. The current density depends therefore on the quotient of effective production and aperture area, which is indirectly proportional to the diameter.

Experimental investigations of aperture diameter variations in monoaperture systems show a similar dependence. [17]

\section{Simulation Results: II. Multiaperture Extraction Systems}

a)

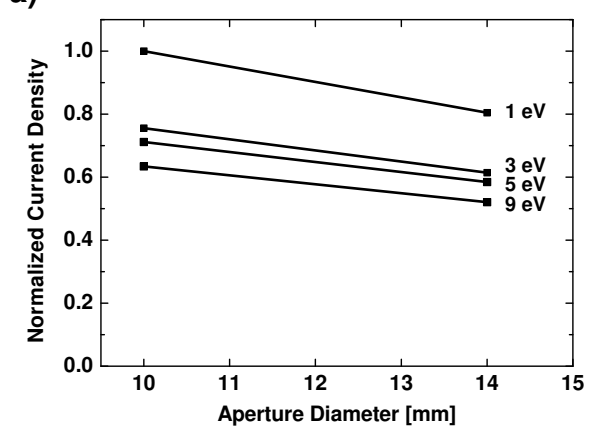

b)

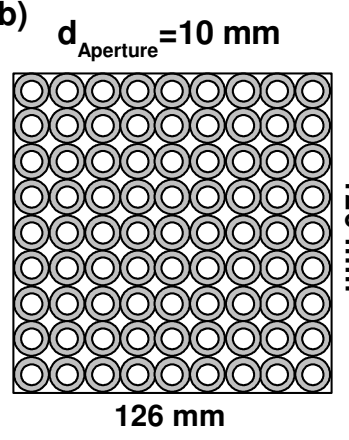

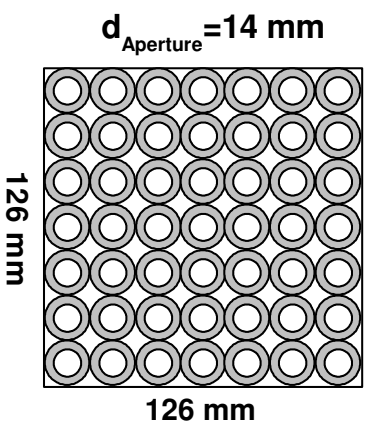

FIGURE 4. a) Normalized multiaperture current density versus the aperture diameter for different starting energies and a constant magnetic field of $8 \mathrm{mT}$. b) Illustration of extractions systems with $10 \mathrm{~mm}$ and $14 \mathrm{~mm}$ aperture diameter. The effective production area is marked in grey.

The effect of the aperture diameter in a multiaperture system is an important question. A converter geometry of a $126 \mathrm{~mm}$ x $126 \mathrm{~mm}$ array of $2 \mathrm{~mm}$ thickness and closely packed, chamfered apertures was investigated for two aperture sizes. Figure 4 b) illustrates these arrays of 49(81) apertures with 14(10) $\mathrm{mm}$ diameter. Similar to the monoaperture calculation, a higher current density is obtained in case of the smaller aperture diameter as illustrated by figure 4 a). An increase of the diameter within the constraints of the defined geometry results in a higher total aperture area, while the effective production area decreases.

Nevertheless, it is not possible to state a simple relation between the current density and the aperture diameter. Additional geometric constraints like the converter thickness and the aperture opening angle have significant influence on the current density by altering the effective production area. A comparison with experimental values requires an exact consideration of these parameters according to the individual geometries of the extraction systems. 


\section{ION BEAM SIMULATION}

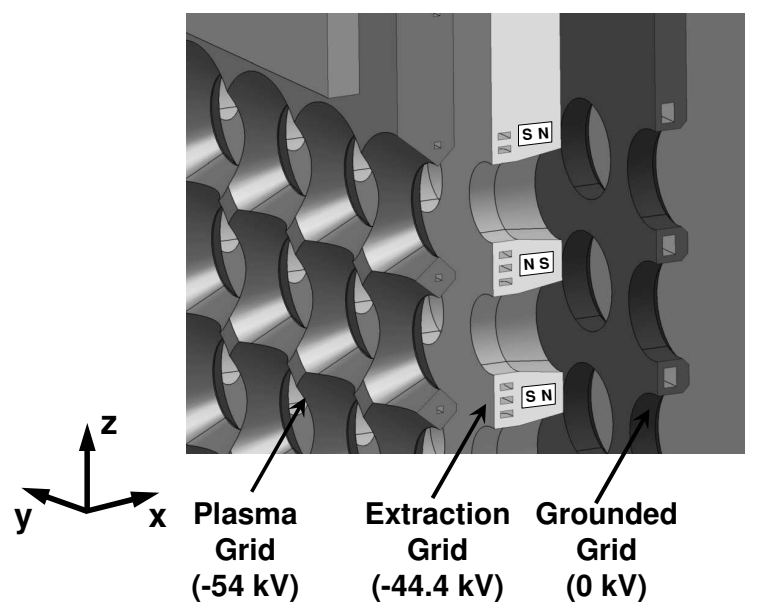

FIGURE 5. Design of the ELISE extraction system including the magnet rods the create the electron deflection field (orthogonal configuration).

A "half-size" ITER-like extraction system is being designed at IPP Garching for the ELISE (Extraction from a Large Ion Source Experiment) [18] test facility.

The ELISE extraction system, based on $14 \mathrm{~mm}$ diameter apertures is shown in figure 5 and will provide an ion acceleration of $60 \mathrm{kV}$. The $\mathrm{x}$-axis is chosen to be the direction of the ion beam acceleration. A periodic arrangement of CoSm magnet rods with alternating magnetization is inserted into the extraction grid to generate the electron deflection field (EDF). This arrangement can be orientated either horizontally (y-direction, as seen in figure 5) or vertically (z-direction). The overall magnetic field results from a superposition with the filter field (FF), which points in the y-direction. This field is produced by an electric current flowing vertically in the PG and by additional permanent magnets positioned on the side of the source vessel. The orientation of the EDF is therefore termed according to the orientation to the FF: orthogonal and parallel.

In order to achieve optimum $\mathrm{Cs}$ conditions, the plasma grid will be operated at $150 \pm 50^{\circ} \mathrm{C}$ [19]. The expansion for the operating range of $\pm 50^{\circ} \mathrm{C}$ causes a maximum aperture offset for the outer most aperture of $0.56 \mathrm{~mm}$ in horizontal direction [18].

Both, the EDF and the plasma grid expansion cause steering effects on the extracted ion beam, which were analyzed by a Ray Tracing simulation.

\section{Simulation Model}

The non-axisymmetric configuration of the electrode geometry owing to the heat induced expansion of the plasma grid and the magnetic field configuration requires a fully $3 \mathrm{~d}$ self consistent calculation of the ion beam formation process. The KOBRA3 code [20] satisfies this criterion. An ANSYS FEM 3d model was used to calculate the magnetic fields in the ELISE extraction system for the ion optics calculation [21]. 


\section{Simulation Results: Beam Steering}

$\mathrm{A} \mathrm{D} \mathrm{D}^{-}$beam with a current density of $260 \mathrm{~A} / \mathrm{m}^{2}$ and an extraction voltage of $9.6 \mathrm{kV}$ was simulated at the outermost aperture in horizontal direction for the orthogonal and parallel EDF configuration. The results of this investigation are given by figure 6 . In the parallel case a), significant beam steering is caused solely by the expansion of the plasma grid in the horizontal direction and is found to be acceptable. The contribution of the FF to the steering is insignificant within the extraction system (domain of $6 \mathrm{~cm}$ ). An intensified steering was observed in case b) of the orthogonal field configuration. In this case, the beam deflection owing to the EDF contributes to the steering by the plasma grid expansion. This results in an intensified steering and leads to minor collisions of the peripheral beam sections with the extraction grid electrode, which is found just acceptable in case of the outermost aperture.
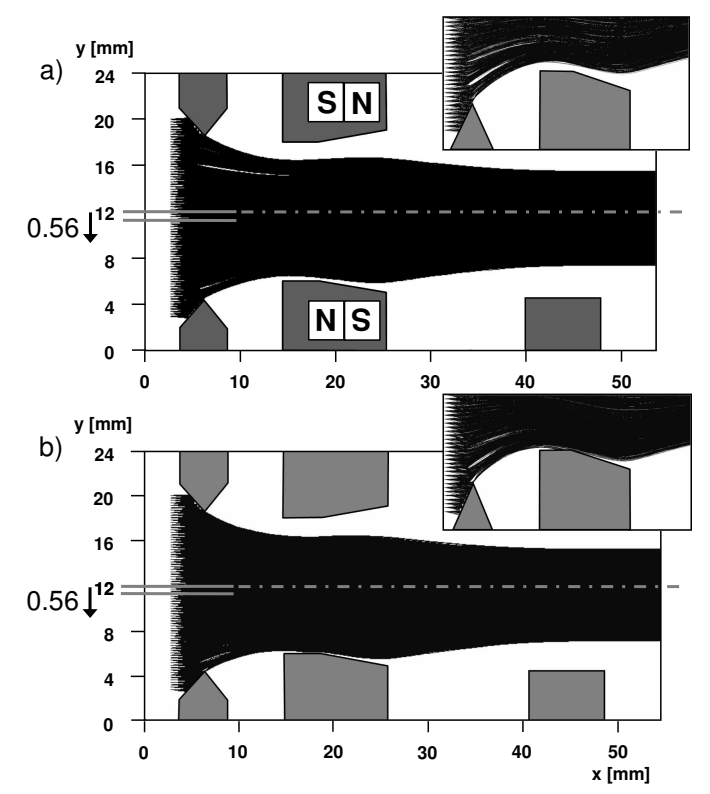

FIGURE 6. Beam steering for the ELISE extraction system for the outermost aperture with a $\mathrm{D}^{-}$current density of $260 \mathrm{~A} / \mathrm{m}^{2}$. a) Filter field and electron deflection field are parallel. b) Filter field and electron deflection field are orthogonal.

\section{CONCLUSION AND OUTLOOK}

Several physical aspects of the generation, transport and extraction of negative ions have been investigated by means of numerical simulations. A calculation of the dynamics of the Cs distribution for vacuum operation based on experimentally determined desorption rates showed, that large amounts of Cs accumulate on the inner walls of the ion source. The influence of Cs poisoning by formation of stable Cs compounds was clarified by comparison of experimental observation with results of the CSFLOW code. Studies of 
the influence of plasma operation on the Cs distribution are in progress.

Ion transport investigations with the TRAJAN code for monoaperture systems showed an indirect proportionality of current density and aperture diameter, that was also observed in the experiment. A relation between the current density and the quotient of effective production and aperture area was found within the scope of the transport simulation. This effective production area is affected by additional geometrical factors, like the converter thickness. Future comparisons with experimental results will therefore include detailed geometric features of the individual extraction systems.

Beam steering effects caused by a combination of the heat load induced plasma grid expansion and the electron deflection field were calculated self-consistently with the KOBRA3 code. An enhanced steering was found in case of the orthogonal magnetic field configuration due to the influence of the electron deflection field. Nevertheless, the steering for the outermost apertures (worst case) was considered just acceptable for both magnetic field configurations.

\section{ACKNOWLEDGMENTS}

This work was (partly) supported by a grant from the European Union within the framework of EFDA (European Fusion Development Agreement). The authors are solely responsible for the content.

\section{REFERENCES}

1. R. S. Hemsworth, A. Tanga and V. Antoni, Rev. Sci. Inst. 79(2008), 02C109

2. E. Speth, H. Falter, P. Franzen, U. Fantz et al, Nucl. Fusion 46 (2006), 220

3. U. Fantz et al., Nucl. Fusion 46 (2006), 297

4. W. G. Graham, "Properties of Alkali Metals Adsorbed onto Metal Surfaces" in Proc. 10th Symp. on Fus. Tech., Brookhaven National Laboratory, Upton, 1980

5. S. Krylov et al., Nucl. Fusion 46 (2006), 324

6. J. D. Levine et al., Surf. Sci. 1 (1964), 171

7. R. Gutser et al., Negative Hydrogen Ion Transport in RF-driven Ion Sources for ITER NBI, to be published

8. O. Fukumasa and R. Nishida, Nucl. Fusion 46, (2006), 275

9. M. Seidl et al, J. Appl. Phys. 79 (1996), 6

10. S. Ma, R. Sydora and J. Dawson, Comp. Phys. Comm. 77 (1993), 190

11. C. Birdsall, IEEE Trans. on Plasma Sci. 19, 1991, 65

12. U. Fantz and D. Wuenderlich, New J. Phys. 8 (2006), 301

13. R. Janev, "Elementary Processes in Hydrogen-Helium Plasmas", Springer, Berlin, 1987

14. M. Eeerden, M. van Sanden, D. Otorbaev and D. Schram, Phys. Rev. A 51 (1994), 3362

15. R. Janev and Z. Radulovic, Phys. Rev. A 17 (1978), 889

16. M. Huels, R. Champion, L. Doverspike and. Y. Wang, Phys. Rev. A 41 (1990), 4809

17. H.P.L. de Esch et al., these proceedings

18. B. Heinemann et al, Design of the "half-size" ITER Neutral Beam Source Test Facility ELISE, SOFT conference 2008, to be published in Fusion Eng. Des.

19. W. Kraus et al., these proceedings

20. P. Spädtke and S. Wipf, GSI Report 89-09 (1989)

21. R. Nocentini, R. Gutser and B. Heinemann, Plasma grid design for optimized filter field configuration for the the NBI test facility ELISE, SOFT conference 2008, to be published in Fusion Eng. Des. 